\title{
A NBEM for the Time-Dependent Anisotropic Problem in an Exterior Elliptic Domain
}

\author{
Qing Chen ${ }^{1}$, Dan Feng ${ }^{1}$, Baoqing Liu $^{2} \&$ Qikui Du ${ }^{1}$ \\ ${ }^{1}$ Jiangsu Key Laboratory for NSLSCS, School of Mathematical Sciences, Nanjing Normal University, Nanjing, \\ China \\ ${ }^{2}$ School of Applied Mathematics, Nanjing University of Finance and Economics, Nanjing, China \\ Correspondence: Qikui Du, School of Mathematical Sciences, Nanjing Normal University, Nanjing 210023, \\ China. E-mail: duqikui@njnu.edu.cn
}

Received: October 8, 2013 Accepted: November 17, 2013 Online Published: November 21, 2013

doi:10.5539/jmr.v5n4p107 URL: http://dx.doi.org/10.5539/jmr.v5n4p107

The research is supported by the National Natural Science Foundation of China, contact/grant number 11371198, 10871100 and 11071109

\begin{abstract}
In this paper, the natural boundary element method (NBEM) for an anisotropic hyperbolic problem in an exterior elliptic domain is investigated. By the theory of the natural boundary reduction (NBR), the natural integral equation (NIE) and the Poisson integral formula of the problem considered are obtained, and the numerical method of the NIE is given. Finally, some numerical examples are presented to demonstrate the performance of the method in the paper.
\end{abstract}

Keywords: anisotropic problem, time-dependent problem, exterior elliptic domain, natural boundary reduction (NBR), numerical solution

\section{Introduction}

In many fields of scientific and engineering computing, these problems in unbounded spatial domains are encountered frequently. The research of numerical solutions for these problems is one of hot spot problems at present. Such problems pose a unique challenge to computation since their domains are unbounded. Although we can apply classical boundary element methods (BEM) or boundary integral methods (BIM) to solve these problems in unbounded domains, a great number of singular integrations usually need to be calculated in practice. Therefore it takes a lot of time to deal with the computation of singular integrations. To overcome some difficulties to solve the problems in unbounded domains numerically, Kang Feng and Dehao Yu have initiated and developed the natural boundary element (NBE) method since the end of 1980's (cf. Feng, 1980, 1983; Yu, 1993, 2002), it is also called as the Dirichlet to Neumann method (DtN method) or the exact artificial boundary condition method (ABCM) lately. NBE method has some distinctive advantages comparing with classical boundary element methods. It is easy to be implemented on the computing, it has good stability of the numerical results, it is fully compatible with finite element method (FEM), and it can be coupled with FEM naturally and directly. For the exterior elliptic problems, the theory of the NBE method is being perfected (cf. Yu, 1993, 2002). For the time-dependent problems, the NBE methods for initial boundary value problems of parabolic and hyperbolic problems are investigated in (cf. Du, 1999, 2001; Hao, 2009). In (Yu, 2003), the coupling of NBE and FEM for the exterior hyperbolic problems is studied.

In this paper, we concentrate on the investigation of the NBE method for the time-dependent anisotropic hyperbolic problem in an exterior elliptic domain by using the similar method as in (Hao, 2009). Using the transformation of variables, we firstly turn the original equation into a Helmholtz equation which is equivalent to the original equation. Then we obtain the Poisson integral formula and the natural integral equation (NIE) of the original problem by using the results in (Zhang, 2008). Finally some numerical examples are presented to illustrate feasibility and efficiency of this method. 
Let $\Omega \subset \mathbb{R}^{2}$ be a bounded simple connected domain with an ellipse $\Gamma \triangleq \partial \Omega$ as its boundary, $\Omega^{c} \triangleq \mathbb{R}^{2} \backslash \bar{\Omega}$. For any fixed positive number $T$, setting $I \triangleq(0, T]$. We consider the following initial-boundary value problems:

$$
\begin{gathered}
\frac{\partial^{2} u}{\partial t^{2}}-\left(a \frac{\partial^{2} u}{\partial x^{2}}+b \frac{\partial^{2} u}{\partial y^{2}}\right)=0, \quad(x, y, t) \in \Omega^{c} \times I, \\
a n_{x} \frac{\partial u}{\partial x}+b n_{y} \frac{\partial u}{\partial y}=g(x, y, t), \quad(x, y, t) \in \Gamma \times I, \\
u(x, y, 0)=u_{0}(x, y), \quad u_{t}(x, y, 0)=v_{0}(x, y), \quad(x, y) \in \Omega^{c},
\end{gathered}
$$

where $a$ and $b$ are all positive constants, $g(x, y, t), u_{0}(x, y)$ and $v_{0}(x, y)$ are all known functions, $\boldsymbol{n}=\left(n_{x}, n_{y}\right)$ is the outer unit normal vector on $\Gamma$ to the internal of $\Omega$. Moreover, we assume that the function $u(x, y, t)$ is bounded at infinity. By the principle of the natural boundary reduction (cf. Yu, 1993, 2002), we have the Poisson integral formula

$$
u(x, y, t)=\mathscr{P} u_{0}(x, y, t), \text { in } \Omega
$$

and the natural integral equation

$$
\frac{\partial u}{\partial n}=\mathscr{K} u_{0}(x, y, t) \text {, on } \Gamma \text {. }
$$

For the sake of convenience, without loss of generality, we assume that $\Omega$ is an ellipse centered at the origin, whose symmetrical axes are $x$-axis and $y$-axis respectively since the expressions of the Poisson integral operator $\mathscr{P}$ and the natural integral operator $\mathscr{K}$ are related to the geometric properties of domain. Moreover, we assume that the solution of the problem considered satisfies appropriate smoothness.

\section{NBR in an Exterior Elliptic Domain}

\subsection{Transformation of Variables}

Let $b>a>0, \Omega^{c}$ be the exterior domain of closed ellipse $\Gamma=\left\{(x, y) \mid \alpha x^{2}+\beta y^{2}=R^{2}\right\}, \boldsymbol{n}=\left(n_{x}, n_{y}\right)=$ $-\left(\frac{\alpha x}{\sqrt{\alpha^{2} x^{2}+\beta^{2} y^{2}}}, \frac{\beta y}{\sqrt{\alpha^{2} x^{2}+\beta^{2} y^{2}}}\right)$. Taking the transformation of variables:

$$
x=\sqrt{a} \xi, \quad y=\sqrt{b} \eta,
$$

thus the closed ellipse $\Gamma$ can be written as $\widetilde{\Gamma}=\left\{(\xi, \eta) \mid a \alpha \xi^{2}+b \beta \eta^{2}=R^{2}\right\}, \Omega^{c}$ is changed into $\widetilde{\Omega^{c}}=\left\{(\xi, \eta) \mid a \alpha \xi^{2}+\right.$ $\left.b \beta \eta^{2}>R^{2}\right\}$. And let

$$
\xi=\frac{1}{\sqrt{a \alpha}} r \cos \phi, \quad \eta=\frac{1}{\sqrt{b \beta}} r \sin \phi
$$

then the outer unit normal vector at the point $(\xi, \eta)$ on $\Gamma$ is

$$
v=-\frac{1}{\sqrt{a \alpha \cos ^{2} \phi+b \beta \sin ^{2} \phi}}(\sqrt{a \alpha} \cos \phi, \sqrt{b \beta} \sin \phi) .
$$

So, the problems (1)-(3) are actually equivalent to the following initial-boundary value problems:

$$
\begin{gathered}
\frac{\partial^{2} u(\xi, \eta, t)}{\partial^{2} t}-\Delta u(\xi, \eta, t)=0, \quad(\xi, \eta, t) \in \widetilde{\Omega^{c}} \times I, \\
\frac{\partial u(\xi, \eta, t)}{\partial n}=\frac{\sqrt{\alpha \cos ^{2} \phi+\beta \sin ^{2} \phi}}{\sqrt{a \alpha \cos ^{2} \phi+b \beta \sin ^{2} \phi}} g(\xi, \eta, t), \quad(\xi, \eta, t) \in \widetilde{\Gamma} \times I, \\
u(\xi, \eta, 0)=u_{0}(\xi, \eta), \quad u_{t}(\xi, \eta, 0)=v_{0}(\xi, \eta), \quad(\xi, \eta) \in \widetilde{\Omega^{c}} .
\end{gathered}
$$

By the separation of variables, the solution of problems (4)-(6) can be expressed as follows:

$$
u(\xi, \eta, t)=Z(\xi, \eta)(\cos \omega t+\sin \omega t), \omega \in \mathbb{R},
$$


then the control equation

$$
\frac{\partial^{2} u(\xi, \eta, t)}{\partial^{2} t}-\Delta u(\xi, \eta, t)=0
$$

can be the Helmholtz as follows:

$$
\Delta Z(\xi, \eta)+\omega^{2} Z(\xi, \eta)=0 .
$$

Introducing elliptic coordinates $(\mu, \phi)$, the relationship between Cartesian coordinates $(\xi, \eta)$ and the elliptic coordinates $(\mu, \phi)$ is as follows (cf. Ben-Poart, 1995; Wu, 2000):

$$
\xi=f_{0} \cosh \mu \cos \phi, \quad \eta=f_{0} \sinh \mu \sin \phi,
$$

where $f_{0}=\sqrt{\frac{b \beta-a \alpha}{a b \alpha \beta}} R, \mu_{0}=\ln \frac{\sqrt{a \alpha}+\sqrt{b \beta}}{\sqrt{b \beta-a \alpha}}, \widetilde{\Gamma}_{\mu_{0}}=\left\{(\mu, \phi) \mid \mu=\mu_{0}, \phi \in[0,2 \pi]\right\}, \widetilde{\Omega}_{c}=\left\{(\mu, \phi) \mid \mu>\mu_{0}, \phi \in\right.$ $[0,2 \pi]\}, \cosh \mu$ and $\sinh \mu$ are the hyperbolic cosine and the hyperbolic sine, respectively. When $\mu$ is given by different values, $\left(f_{0}, 0\right),\left(-f_{0}, 0\right)$ are the co-foci of ellipse families $\widetilde{\Gamma}_{\mu}$.

If let $J(\mu, \phi)=\left|\begin{array}{cc}\xi_{\mu} & \xi_{\phi} \\ \eta_{\mu} & \eta_{\phi}\end{array}\right|$, then $J(\mu, \phi)=f_{0}^{2} \cosh ^{2} \mu \sin ^{2} \phi+f_{0}^{2} \sinh ^{2} \mu \cos ^{2} \phi$. It is not difficult to obtain that

$$
J\left(\mu_{0}, \phi\right)=\frac{R^{2}}{a b \alpha \beta}\left(b \beta \sin ^{2} \phi+a \alpha \cos ^{2} \phi\right) .
$$

Lemma 2.1 (Wu, 2000; Zhu, 2004) Transformation (8) has the following properties:

(i) The Jacobi determination of the transformation (8)

$$
J(\mu, \phi)=f_{0}^{2} \cosh ^{2} \mu \sin ^{2} \phi+f_{0}^{2} \sinh ^{2} \mu \cos ^{2} \phi=f_{0}^{2}\left(\cosh ^{2} \mu-\cos ^{2} \phi\right),
$$

$J(\mu, \phi)=0$ if and only if $(\xi, \eta)=\left( \pm f_{0}, 0\right)$;

(ii) For $u \in C^{2}\left(\mathbb{R}^{2}\right)$, the following holds

$$
\frac{\partial^{2} u}{\partial \mu^{2}}+\frac{\partial^{2} u}{\partial \phi^{2}}=J(\mu, \phi)\left(\frac{\partial^{2} u}{\partial \xi^{2}}+\frac{\partial^{2} u}{\partial \eta^{2}}\right)
$$

(iii) Let $\Gamma_{\mu_{1}} \triangleq\left\{(\mu, \phi) \mid \mu=\mu_{1}, \phi \in[0,2 \pi]\right\}$ be the inner boundary $\Omega_{\mu_{1}}^{c} \triangleq\left\{(\mu, \phi) \mid \mu>\mu_{1}, \phi \in[0,2 \pi]\right\}, v$ be the outer unit normal vector on $\Gamma_{\mu_{1}}$, then

$$
\frac{\partial u}{\partial v}=-\frac{1}{\sqrt{J\left(\mu_{1}, \phi\right)}} \frac{\partial u}{\partial \mu}
$$

Under the elliptic coordinates, Equation (7) can be expressed as follows:

$$
\frac{1}{J(\mu, \phi)}\left(\frac{\partial^{2} Z}{\partial \mu^{2}}+\frac{\partial^{2} Z}{\partial \phi^{2}}\right)+\omega^{2} Z=0
$$

By the separation of variables, we know that the solution of the problem (13) can be expressed as $Z(\mu, \phi)=$ $F(\mu) G(\phi)$, and $F(\mu), G(\phi)$ satisfy

$$
\begin{gathered}
G^{\prime \prime}(\phi)+(p-2 q \cos 2 \phi) G(\phi)=0, \\
F^{\prime \prime}(\mu)+(p-2 q \cosh 2 \mu) F(\mu)=0,
\end{gathered}
$$

where $p=k-\frac{\alpha^{2}}{2}, q=\frac{\alpha^{2}}{4}, \alpha^{2}=\omega^{2} f_{0}^{2}, k$ is a parameter. From Wang and Guo (1979), we know that Equations (14) and (15) are the Mathieu and the modified Mathieu equation, respectively. Their solutions are the angular Mathieu functions (AMF) and the radial Mathieu functions (RMF), $0 \leqslant \phi<2 \pi, 0 \leqslant \mu<\infty$.

\subsection{Angular Mathieu Functions and Radial Mathieu Functions}

Angular Mathieu equation is a second-order linear differential equation, it has two linearly independent solutions, which are called as the even Mathieu functions and the odd Mathieu functions

$$
\Phi_{m}(\phi ; q)= \begin{cases}c e_{m}(\phi ; q), & m=0,1,2, \cdots \\ s e_{m}(\phi ; q), & m=1,2,3, \cdots,\end{cases}
$$


here $m$ is the order of Mathieu equation. The period of $\Phi_{m}(\phi ; q)$ is $\pi$ when $m$ is even, the period of $\Phi_{m}(\phi ; q)$ is $2 \pi$ when $m$ is odd number. The number $p$ in Equations (14) and (15) denotes eigenvalues of even and odd angular Mathieu functions, respectively. For the computation of the even Mathieu function $c e_{m}(\varphi, q)$ and the odd Mathieu function $\operatorname{se}_{m}(\phi ; q)$ can refer to (Hao, 2009).

When $q$ is positive, the solutions of Equation (15) are called as the even radial Mathieu functions of the first kind and the odd radial Mathieu functions of the second kind

$$
R_{m}(\mu ; q)= \begin{cases}J e_{m}(\mu ; q), & J o_{m}(\mu ; q) \text { (the first kind), } \\ N e_{m}(\mu ; q), & N o_{m}(\mu ; q) \text { (the second kind), }\end{cases}
$$

here $m$ is the order of the modified Mathieu function.

For the modified Mathieu functions, we can define (Hao, 2009)

$$
\begin{gathered}
M c_{2 n}^{(j)}(\mu ; q)=\sum_{\ell=0}^{\infty}(-1)^{\ell+n} \frac{A_{2 \ell}^{2 n}}{\left(A_{0}^{2 n}\right)^{-1}} J_{\ell}\left(u_{1}\right) Z_{\ell}^{(j)}\left(u_{2}\right), \\
M c_{2 n+1}^{(j)}(\mu ; q)=\sum_{\ell=0}^{\infty}(-1)^{\ell+n} \frac{A_{2 \ell+1}^{2 n+1}}{\left(A_{1}^{2 n+1}\right)^{-1}}\left[J_{\ell}\left(u_{1}\right) Z_{\ell+1}^{(j)}\left(u_{2}\right)+J_{\ell+1}\left(u_{1}\right) Z_{\ell}^{(j)}\left(u_{2}\right)\right], \\
M s_{2 n+1}^{(j)}(\mu ; q)=\sum_{\ell=0}^{\infty}(-1)^{\ell+n} \frac{B_{2 \ell+1}^{2 n+1}}{\left(B_{1}^{2 n+1}\right)^{-1}}\left[J_{\ell}\left(u_{1}\right) Z_{\ell+1}^{(j)}\left(u_{2}\right)-J_{\ell+1}\left(u_{1}\right) Z_{\ell}^{(j)}\left(u_{2}\right)\right], \\
M s_{2 n+2}^{(j)}(\mu ; q)=\sum_{\ell=0}^{\infty}(-1)^{\ell+n} \frac{B_{2 \ell+2}^{2 n+2}}{\left(B_{2}^{2 n+2}\right)^{-1}}\left[J_{\ell}\left(u_{1}\right) Z_{\ell+2}^{(j)}\left(u_{2}\right)-J_{\ell+2}\left(u_{1}\right) Z_{\ell}^{(j)}\left(u_{2}\right)\right],
\end{gathered}
$$

where $j=1,2,3,4, Z_{\ell}^{(1)}(x)=J_{\ell}(x), Z_{\ell}^{(2)}(x)=Y_{\ell}(x), Z_{\ell}^{(3)}(x)=H_{\ell}^{(1)}(x), Z_{\ell}^{(4)}(x)=H_{\ell}^{(2)}(x), u_{1}=\sqrt{q} \exp (-\mu)$, $u_{2}=\sqrt{q} \exp (\mu) . M c_{m}^{(j)}(\mu ; q)$ and $M s_{m}^{(j)}(\mu ; q)$ are the even and odd the modified Mathieu functions of the j-th kind. The expansion coefficients and eigenvalues of the modified Mathieu functions are same as the ones of the Mathieu functions.

\subsection{The Poisson Integral Formula and the Natural Integral Equation}

For the exterior problem, the solutions of Equation (15) can expressed by the even modified Mathieu functions of the first kind and the second kind

$$
H o_{m}(\mu ; q)=J o_{m}(\mu ; q)+i N o_{m}(\mu ; q), H e_{m}(\mu ; q)=J e_{m}(\mu ; q)+i N e_{m}(\mu ; q)
$$

Hence, under the elliptic coordinates the solutions of Equations (4)-(6) can be expressed as

$$
u e_{m}=H e_{m}(\mu) c e_{m}(\phi, q)[\cos (\omega t)+\sin (\omega t)], u o_{m}=H o_{m}(\mu) s e_{m}(\phi, q)[\cos (\omega t)+\sin (\omega t)]
$$

For the even functions $u e_{m}$, we have $m \geqslant 0$, while the odd function $u o_{m}$, we have $m \geqslant 1$. Let $H o_{0}=s e_{0}=0$, so

$$
u(\mu, \phi, t)=\sum_{m=0}^{\infty}\left[C_{m}(t) H e_{m}(\mu) c e_{m}(\phi, q)+D_{m}(t) H o_{m}(\mu) s e_{m}(\phi, q)\right](\cos (\omega t)+\sin (\omega t)), \quad \mu>\mu_{0},
$$

where $C_{m}$ and $D_{m}$ are some constants. From (22), we have

$$
u_{0}\left(\mu_{0}, \phi, t\right)=\sum_{m=0}^{\infty}\left[C_{m}(t) H e_{m}\left(\mu_{0}\right) c e_{m}(\phi, q)+D_{m}(t) H o_{m}\left(\mu_{0}\right) s e_{m}(\phi, q)\right](\cos (\omega t)+\sin (\omega t)) .
$$

Using these above, we can obtain

$$
\begin{gathered}
C_{m}(t)=\frac{1}{\pi H e_{m}\left(\mu_{0}\right)(\cos (\omega t)+\sin (\omega t))} \int_{0}^{2 \pi} u\left(\mu_{0}, \phi^{\prime}, t\right) c e_{m}\left(\phi^{\prime}, q\right) d \phi^{\prime}, \quad m=0,1, \cdots, \\
D_{m}(t)=\frac{1}{\pi H o_{m}\left(\mu_{0}\right)(\cos (\omega t)+\sin (\omega t))} \int_{0}^{2 \pi} u\left(\mu_{0}, \phi^{\prime}, t\right) s e_{m}\left(\phi^{\prime}, q\right) d \phi^{\prime}, \quad m=0,1, \cdots .
\end{gathered}
$$


Substituting $C_{m}$ and $D_{m}$ to (22), the function $u(\mu, \phi, t)$ can be expressed as follows:

$$
\begin{aligned}
u(\mu, \phi, t)= & \frac{1}{\pi} \sum_{m=0}^{\infty} \int_{0}^{2 \pi}\left[\frac{H e_{m}(\mu)}{H e_{m}\left(\mu_{0}\right)} c e_{m}(\phi, q) c e_{m}\left(\phi^{\prime}, q\right)\right. \\
& \left.+\frac{H o_{m}(\mu)}{H o_{m}\left(\mu_{0}\right)} \operatorname{se}_{m}(\phi, q) s e_{m}\left(\phi^{\prime}, q\right)\right] u_{0}\left(\mu_{0}, \phi^{\prime}, t\right) d \phi^{\prime} \triangleq \mathscr{P} u_{0}\left(\mu_{0}, \phi^{\prime}, t\right), \mu>\mu_{0} .
\end{aligned}
$$

(23) is the Poisson integral formula. Taking the derivatives of function $u(\mu, \phi, t)$ with respect to $\mu$, and taking the limitation as $\mu$ approaches to $\mu_{0}$, we have

$$
\begin{aligned}
\frac{\partial u}{\partial v}= & -\frac{1}{\pi \sqrt{J\left(\mu_{0}, \phi\right)}} \sum_{m=0}^{\infty} \int_{0}^{2 \pi}\left[\frac{H e_{m}^{\prime}\left(\mu_{0}\right)}{H e_{m}\left(\mu_{0}\right)} c e_{m}(\phi, q) c e_{m}\left(\phi^{\prime}, q\right)\right. \\
& \left.+\frac{H o_{m}^{\prime}\left(\mu_{0}\right)}{H o_{m}\left(\mu_{0}\right)} s e_{m}(\phi, q) s e_{m}\left(\phi^{\prime}, q\right)\right] u_{0}\left(\mu_{0}, \phi^{\prime}, t\right) d \phi^{\prime} \triangleq \mathscr{K} u_{0}\left(\mu_{0}, \phi^{\prime}, t\right), \mu=\mu_{0} .
\end{aligned}
$$

Here $H e_{m}^{\prime}\left(\mu_{0}\right)$ and $H o_{m}^{\prime}\left(\mu_{0}\right)$ are the derivatives of functions $H e_{m}(\mu)$ and $H o_{m}(\mu)$ at $\mu=\mu_{0}$. (24) is the natural integral equation of the problems (4)-(6), and $\mathscr{K}$ is called as the natural integral operator (NBO). If (24) is solved, the solution of the problems (4)-(6) can be obtained, and we can obtain the solution of the problems (1)-(3).

\subsection{Direct Investigation of the NBO}

Mathieu functions $c e_{m}(\phi, q)$ and $s e_{m}(\phi, q)$ satisfy the following orthogonal relations

$$
\int_{0}^{2 \pi} c e_{m}(\phi, q) c e_{n}(\phi, q) d \phi=\int_{0}^{2 \pi} s e_{m}(\phi, q) s e_{n}(\phi, q) d \phi= \begin{cases}\pi, & m=n, \\ 0, & m \neq n .\end{cases}
$$

Similar to the expansion of Fourier series, for any $v \in H^{s}(\widetilde{\Gamma})$, it can be expressed as

$$
v=\frac{1}{\pi} \sum_{m=0}^{\infty}\left(\int_{0}^{2 \pi} c e_{m}(\phi, q) v d \phi\right) c e_{m}(\phi, q)+\left(\int_{0}^{2 \pi} s e_{m}(\phi, q) v d \phi\right) s e_{m}(\phi, q) .
$$

From $\left\langle c e_{m}(\phi, q), v\right\rangle=\int_{0}^{2 \pi} c e_{m}(\phi, q) v d s=\int_{0}^{2 \pi} c e_{m}(\phi, q) v \sqrt{J\left(\mu_{0}, \phi\right)} d \phi$, we have

$$
\left\langle v, \frac{c e_{m}(\phi, q)}{\sqrt{J\left(\mu_{0}, \phi\right)}}\right\rangle_{\widetilde{\Gamma}}=\int_{0}^{2 \pi} c e_{m}(\phi, q) v d \phi
$$

Thus, $v$ can be rewritten as

$$
v=\frac{1}{\pi} \sum_{m=0}^{\infty}\left\langle v, \frac{c e_{m}(\phi, q)}{\sqrt{J\left(\mu_{0}, \phi\right)}}\right\rangle_{\Gamma} c e_{m}(\phi, q)+\left\langle v, \frac{s e_{m}(\phi, q)}{\sqrt{J\left(\mu_{0}, \phi\right)}}\right\rangle_{\widetilde{\Gamma}} s e_{m}(\phi, q) .
$$

To cope with the numerical analysis we recall an equivalent definition of the norm of Sobolev space $H^{s}(\widetilde{\Gamma})$ for any real number $s$ :

$$
\|v\|_{s, \widetilde{\Gamma}}^{2} \triangleq \frac{1}{\pi^{2}} \sum_{m=0}^{+\infty}\left(1+m^{2}\right)^{s}\left[\left|\left\langle v, \frac{c e_{m}(\phi, q)}{\sqrt{J\left(\mu_{0}, \phi\right)}}\right\rangle_{\widetilde{\Gamma}}\right|^{2}+\left|\left\langle v, \frac{s e_{m}(\phi, q)}{\sqrt{J\left(\mu_{0}, \phi\right)}}\right\rangle_{\widetilde{\Gamma}}\right|^{2}\right] .
$$

Theorem 2.2 An operator $\mathscr{K}: H^{\frac{1}{2}}(\widetilde{\Gamma}) \rightarrow H^{-\frac{1}{2}}(\widetilde{\Gamma})$ is linear continuous, that is, there exists a positive constant $C$ such that

$$
\|\mathscr{K} f\|_{-\frac{1}{2}, \widetilde{\Gamma}} \leqslant C\|f\|_{\frac{1}{2}, \widetilde{\Gamma}}, \quad \forall f \in H^{\frac{1}{2}}(\widetilde{\Gamma}) .
$$

Proof. It is easy to know that natural integral operator $\mathscr{K}$ is a linear operator. For any $f(\phi) \in H^{\frac{1}{2}}(\widetilde{\Gamma})$, it is expressed by the following series

$$
f=\frac{1}{\pi} \sum_{m=0}^{\infty}\left\langle f, \frac{c e_{m}(\phi, q)}{\sqrt{J\left(\mu_{0}, \phi\right)}}\right\rangle_{\widetilde{\Gamma}} c e_{m}(\phi, q)+\left\langle f, \frac{s e_{m}(\phi, q)}{\sqrt{J\left(\mu_{0}, \phi\right)}}\right\rangle_{\widetilde{\Gamma}} s e_{m}(\phi, q) .
$$


Since

$$
\begin{gathered}
\|f\|_{s, \widetilde{\Gamma}}^{2}=\frac{1}{\pi^{2}} \sum_{m=0}^{+\infty}\left(1+m^{2}\right)^{\frac{1}{2}}\left[\left|\left\langle f, \frac{c e_{m}(\phi, q)}{\sqrt{J\left(\mu_{0}, \phi\right)}}\right\rangle_{\Gamma}\right|^{2}+\left|\left\langle f, \frac{s e_{m}(\phi, q)}{\sqrt{J\left(\mu_{0}, \phi\right)}}\right\rangle_{\Gamma}\right|^{2}\right], \\
\mathscr{K} f(\phi)=-\frac{1}{\pi \sqrt{J\left(\mu_{0}, \phi\right)}} \sum_{m=0}^{\infty} \int_{0}^{2 \pi}\left[\frac{H e_{m}^{\prime}\left(\mu_{0}\right)}{H e_{m}\left(\mu_{0}\right)} c e_{m}(\phi, q) c e_{m}\left(\phi^{\prime}, q\right)+\frac{H o_{m}^{\prime}\left(\mu_{0}\right)}{H o_{m}\left(\mu_{0}\right)} s e_{m}(\phi, q) s e_{m}\left(\phi^{\prime}, q\right)\right] f\left(\mu_{0}, \phi^{\prime}, t\right) d \phi^{\prime} \\
=\frac{1}{\pi \sqrt{J\left(\mu_{0}, \phi\right)}} \sum_{m=0}^{\infty}\left[-\frac{H e_{m}^{\prime}\left(\mu_{0}\right)}{H e_{m}\left(\mu_{0}\right)}\left\langle f, \frac{c e_{m}(\phi, q)}{\sqrt{J\left(\mu_{0}, \phi\right)}}\right\rangle_{\widetilde{\Gamma}} c e_{m}(\phi, q)-\frac{H o_{m}^{\prime}\left(\mu_{0}\right)}{H o_{m}\left(\mu_{0}\right)}\left\langle f, \frac{s e_{m}(\phi, q)}{\sqrt{J\left(\mu_{0}, \phi\right)}}\right\rangle_{\widetilde{\Gamma}} s e_{m}(\phi, q)\right] .
\end{gathered}
$$

So

$$
\|\mathscr{K} f(\phi)\|_{-\frac{1}{2}, \widetilde{\Gamma}}^{2}=\frac{1}{\pi^{2} J\left(\mu_{0}, \phi\right)} \sum_{m=0}^{\infty}\left(1+m^{2}\right)^{-\frac{1}{2}}\left[\left|-\frac{H e_{m}^{\prime}\left(\mu_{0}\right)}{H e_{m}\left(\mu_{0}\right)}\left\langle f, \frac{c e_{m}(\phi, q)}{\sqrt{J\left(\mu_{0}, \phi\right)}}\right\rangle_{\Gamma}\right|^{2}+\left|-\frac{H o_{m}^{\prime}\left(\mu_{0}\right)}{H o_{m}\left(\mu_{0}\right)}\left\langle f, \frac{s e_{m}(\phi, q)}{\sqrt{J\left(\mu_{0}, \phi\right)}}\right\rangle_{\Gamma}\right|^{2}\right] .
$$

A computation shows that

$$
\frac{H e_{m}^{\prime}\left(\mu_{0}, q\right)}{H e_{m}\left(\mu_{0}, q\right)} \approx \alpha\left(1+m^{2}\right)^{\frac{1}{2}}, \frac{H o_{m}^{\prime}\left(\mu_{0}, q\right)}{H o_{m}\left(\mu_{0}, q\right)} \approx \alpha\left(1+m^{2}\right)^{\frac{1}{2}}, \alpha \in\left(-2,-\frac{1}{2}\right)
$$

Therefore

$$
\begin{aligned}
\|\mathscr{K} f(\phi)\|_{-\frac{1}{2}, \widetilde{\Gamma}}^{2} & \leqslant \frac{4}{\pi^{2} J\left(\mu_{0}, \phi\right)} \sum_{m=0}^{\infty}\left(1+m^{2}\right)^{\frac{1}{2}}\left[\left|\left\langle f, \frac{c e_{m}(\phi, q)}{\sqrt{J\left(\mu_{0}, \phi\right)}}\right\rangle_{\widetilde{\Gamma}}\right|^{2}+\left|\left\langle f, \frac{s e_{m}(\phi, q)}{\sqrt{J\left(\mu_{0}, \phi\right)}}\right\rangle_{\widetilde{\Gamma}}\right|^{2}\right] \\
& =\frac{4}{J\left(\mu_{0}, \phi\right)}\|f\|_{s, \widetilde{\Gamma}}^{2} .
\end{aligned}
$$

Noting that

$$
J\left(\mu_{0}, \phi\right)=\frac{R^{2}}{a b}\left(b \sin ^{2} \phi+a \cos ^{2} \phi\right)=\frac{R^{2}}{a b}\left(\frac{a+b}{2}+\frac{a-b}{2} \cos 2 \phi\right), \frac{R^{2}}{b} \leqslant J\left(\mu_{0}, \phi\right) \leqslant \frac{R^{2}}{a},
$$

thus $\frac{a}{R^{2}} \leqslant \frac{1}{J\left(\mu_{0}, \phi\right)} \leqslant \frac{b}{R^{2}}$. This proves that $\|\mathscr{K} f(\phi)\|_{-\frac{1}{2}, \widetilde{\Gamma}}^{2} \leqslant \frac{4 \sqrt{b}}{R}\|f\|_{s, \widetilde{\Gamma}}^{2}$.

\section{Numerical Solutions of the NIE}

Let $\tau$ be the time-step interval, and $t_{k}=k \tau, v^{k}(\mu, \phi)=v\left(\mu, \phi, t_{k}\right), k=1,2, \ldots, N_{0}, N_{0}=[T / \tau]$. Then the semidiscrete formulation of (23) and (24), respectively, as follows

$$
\begin{gathered}
u^{k}(\mu, \phi)=\frac{1}{\pi} \sum_{m=0}^{\infty} \int_{0}^{2 \pi}\left[\frac{H e_{m}(\mu)}{H e_{m}\left(\mu_{0}\right)} c e_{m}(\phi, q) c e_{m}\left(\phi^{\prime}, q\right)+\frac{H o_{m}(\mu)}{H o_{m}\left(\mu_{0}\right)} s e_{m}(\phi, q) s e_{m}\left(\phi^{\prime}, q\right)\right] u_{0}^{k}\left(\mu_{0}, \phi^{\prime}\right) d \phi^{\prime}, \mu>\mu_{0}, \\
g^{k}\left(\mu_{0}, \phi\right)=-\frac{\sqrt{a b \alpha \beta}}{R \pi \cdot \sqrt{\alpha \cos ^{2} \phi+\beta \sin ^{2} \phi}} \sum_{m=0}^{\infty} \int_{0}^{2 \pi}\left[\frac{H e_{m}^{\prime}\left(\mu_{0}\right)}{H e_{m}\left(\mu_{0}\right)} c e_{m}(\phi, q) c e_{m}\left(\phi^{\prime}, q\right)\right. \\
\left.+\frac{H o_{m}^{\prime}\left(\mu_{0}\right)}{H o_{m}\left(\mu_{0}\right)} \operatorname{se}_{m}(\phi, q) \operatorname{se}_{m}\left(\phi^{\prime}, q\right)\right] u_{0}^{k}\left(\mu_{0}, \phi^{\prime}\right) d \phi^{\prime} \triangleq \mathscr{K}^{k} u_{0}^{k}\left(\mu_{0}, \phi^{\prime}\right) .
\end{gathered}
$$

For (26), we have the following variational problem:

$$
\text { Find } u_{0}^{k}(\mu, \phi) \in H^{\frac{1}{2}}(\widetilde{\Gamma}) \text {, such that } b\left(u_{0}^{k}, v^{k}\right)=\left\langle g^{k}, v^{k}\right\rangle, \forall v^{k} \in H^{\frac{1}{2}}(\widetilde{\Gamma}),
$$

where

$$
b\left(u_{0}^{k}, v^{k}\right)=\left\langle\mathscr{K}^{k} u_{0}^{k}, v^{k}\right\rangle=\int_{\widetilde{\Gamma}}\left(\mathscr{K}^{k} u_{0}^{k}\left(\mu_{0}, \phi\right)\right) \cdot v^{k} d s,\left\langle g^{k}, v^{k}\right\rangle=\int_{\widetilde{\Gamma}} g^{k} v^{k} d s .
$$

Theorem 3.1 The bilinear form $b(\cdot, \cdot)$, which is defined by the natural integral operator $\mathscr{K}$, is symmetric and continuous on $H^{\frac{1}{2}}(\widetilde{\Gamma}) \times H^{\frac{1}{2}}(\widetilde{\Gamma})$, and $V$-elliptic. 
Proof. From the definition of the bilinear form $b(\cdot, \cdot)$, we have

$$
\begin{aligned}
b(u, v)= & \int_{\widetilde{\Gamma}}-\frac{1}{\pi \sqrt{J\left(\mu_{0}, \phi\right)}} \sum_{m=0}^{\infty} \int_{0}^{2 \pi}\left[\frac{H e_{m}^{\prime}\left(\mu_{0}\right)}{H e_{m}\left(\mu_{0}\right)} c e_{m}(\phi, q) c e_{m}\left(\phi^{\prime}, q\right)\right] \\
& \left.+\frac{H o_{m}^{\prime}\left(\mu_{0}\right)}{H o_{m}\left(\mu_{0}\right)} s e_{m}(\phi, q) s e_{m}\left(\phi^{\prime}, q\right)\right] u\left(\mu_{0}, \phi^{\prime}, t\right) v\left(\mu_{0}, \phi, t\right) d \phi^{\prime} d s \\
= & -\frac{1}{\pi} \int_{0}^{2 \pi} \int_{0}^{2 \pi} \sum_{m=0}^{\infty}\left[\frac{H e_{m}^{\prime}\left(\mu_{0}\right)}{H e_{m}\left(\mu_{0}\right)} c e_{m}(\phi, q) c e_{m}\left(\phi^{\prime}, q\right)\right] \\
& \left.+\frac{H o_{m}^{\prime}\left(\mu_{0}\right)}{H o_{m}\left(\mu_{0}\right)} s e_{m}(\phi, q) s e_{m}\left(\phi^{\prime}, q\right)\right] u\left(\mu_{0}, \phi^{\prime}, t\right) v\left(\mu_{0}, \phi, t\right) d \phi^{\prime} d \phi .
\end{aligned}
$$

Hence, $b(u, v)=b(v, u)$, this proves $b(\cdot, \cdot)$ is symmetric on $H^{\frac{1}{2}}(\widetilde{\Gamma}) \times H^{\frac{1}{2}}(\widetilde{\Gamma})$.

Using Theorem 2.2, for any $u, v \in H^{\frac{1}{2}}(\widetilde{\Gamma})$, we have

$$
b(u, v)=\langle\mathscr{K} u, v\rangle \leqslant\|\mathscr{K} u\|_{-\frac{1}{2}, \widetilde{\Gamma}}\|v\|_{\frac{1}{2}, \widetilde{\Gamma}} \leqslant C\|u\|_{\frac{1}{2}, \widetilde{\Gamma}}\|v\|_{\frac{1}{2}, \widetilde{\Gamma}},
$$

this proves $b(\cdot, \cdot)$ is continuous on $H^{\frac{1}{2}}(\widetilde{\Gamma}) \times H^{\frac{1}{2}}(\widetilde{\Gamma})$. For any $u \in H^{\frac{1}{2}}(\widetilde{\Gamma})$, we have

$$
\begin{aligned}
& b(u, u)=\langle\mathscr{K} u, u\rangle=-\frac{1}{\pi} \int_{0}^{2 \pi} \int_{0}^{2 \pi} \sum_{m=0}^{\infty}\left[\frac{H e_{m}^{\prime}\left(\mu_{0}\right)}{H e_{m}\left(\mu_{0}\right)} c e_{m}(\phi, q) c e_{m}\left(\phi^{\prime}, q\right)\right. \\
& \left.+\frac{H o_{m}^{\prime}\left(\mu_{0}\right)}{H o_{m}\left(\mu_{0}\right)} s e_{m}(\phi, q) s e_{m}\left(\phi^{\prime}, q\right)\right] u\left(\mu_{0}, \phi^{\prime}, t\right) u\left(\mu_{0}, \phi, t\right) d \phi^{\prime} d \phi \\
& =\frac{1}{\pi} \sum_{m=0}^{\infty}\left[-\frac{H e_{m}^{\prime}\left(\mu_{0}\right)}{H e_{m}\left(\mu_{0}\right)}\left\langle u, \frac{c e_{m}(\phi, q)}{\sqrt{J\left(\mu_{0}, \phi\right)}}\right\rangle_{\widetilde{\Gamma}}\left\langle u, \frac{c e_{m}\left(\phi^{\prime}, q\right)}{\sqrt{J\left(\mu_{0}, \phi^{\prime}\right)}}\right\rangle_{\widetilde{\Gamma}}\right. \\
& \left.-\frac{H o_{m}^{\prime}\left(\mu_{0}\right)}{H o_{m}\left(\mu_{0}\right)}\left\langle u, \frac{s e_{m}(\phi, q)}{\sqrt{J\left(\mu_{0}, \phi\right)}}\right\rangle_{\widetilde{\Gamma}}\left\langle u, \frac{s e_{m}\left(\phi^{\prime}, q\right)}{\sqrt{J\left(\mu_{0}, \phi^{\prime}\right)}}\right\rangle_{\widetilde{\Gamma}}\right] \\
& =\frac{1}{\pi} \sum_{m=0}^{\infty}\left[-\frac{H e_{m}^{\prime}\left(\mu_{0}\right)}{H e_{m}\left(\mu_{0}\right)}\left|\left\langle u, \frac{c e_{m}(\phi, q)}{\sqrt{J\left(\mu_{0}, \phi\right)}}\right\rangle\right|_{\widetilde{\Gamma}}^{2}-\frac{H o_{m}^{\prime}\left(\mu_{0}\right)}{H o_{m}\left(\mu_{0}\right)}\left|\left\langle u, \frac{s e_{m}(\phi, q)}{\sqrt{J\left(\mu_{0}, \phi\right)}}\right\rangle_{\Gamma}\right|^{2}\right] \\
& \frac{1}{2 \pi} \sum_{m=0}^{\infty}\left(1+m^{2}\right)^{\frac{1}{2}}\left[\left|\left\langle u, \frac{c e_{m}(\phi, q)}{\sqrt{J\left(\mu_{0}, \phi\right)}}\right\rangle\right|_{\widetilde{\Gamma}}^{2}+\left|\left\langle u, \frac{s e_{m}(\phi, q)}{\sqrt{J\left(\mu_{0}, \phi\right)}}\right\rangle_{\widetilde{\Gamma}}\right|^{2}\right] \\
& \geqslant \frac{1}{\pi^{2}} \sum_{m=0}^{\infty}\left(1+m^{2}\right)^{\frac{1}{2}}\left[\left|\left\langle u, \frac{c e_{m}(\phi, q)}{\sqrt{J\left(\mu_{0}, \phi\right)}}\right\rangle_{\widetilde{\Gamma}}\right|^{2}+\left|\left\langle u, \frac{s e_{m}(\phi, q)}{\sqrt{J\left(\mu_{0}, \phi\right)}}\right\rangle_{\widetilde{\Gamma}}\right|^{2}\right]=\|u\|_{\frac{1}{2}, \widetilde{\Gamma}}^{2} .
\end{aligned}
$$

\subsection{Discretization and Computation of Stiffness Matrices}

To solve the variational problem (27), we now divide the interval $[0,2 \pi]$ into $N$ sub-intervals, Setting $h=2 \pi / N$, and let $S^{h}(\widetilde{\Gamma})$ is the finite element subspace of $H^{\frac{1}{2}}(\widetilde{\Gamma})$. Then the problem of (27) can be written as

$$
\text { Find } u_{0 h}^{k}(\mu, \phi) \in S^{h}(\widetilde{\Gamma}) \text {, such that } b\left(u_{0 h}^{k}, v^{k}\right)=\left\langle g^{k}, v^{k}\right\rangle, \quad \forall v^{k} \in S^{h}(\widetilde{\Gamma}) \text {. }
$$

Let $\varphi_{k}(\phi), k=1,2, \ldots, N$ be base functions of $S^{h}(\widetilde{\Gamma})$, then $u_{0 h}^{k}\left(\mu_{0}, \phi\right)$ can be expressed as $u_{0 h}^{k}\left(\mu_{0}, \phi\right)=\sum_{j=1}^{N} u_{0 j}^{k} \varphi_{j}(\phi)$.

Substituting it into (28), we easily obtain the system of linear algebraic equations of (28) as follows:

$$
\boldsymbol{Q U} \boldsymbol{U}^{k}=\boldsymbol{b}^{k}
$$

where

$$
\begin{gathered}
\boldsymbol{Q}=\left(q_{i j}\right)_{N \times N}, \boldsymbol{U}^{k}=\left(u_{01}^{k}, u_{02}^{k}, \ldots, u_{0 N}^{k}\right)^{T}, \\
\boldsymbol{b}^{k}=\left(b_{1}^{k}, b_{2}^{k}, \ldots, b_{N}^{k}\right)^{T}, b_{j}^{k}=\int_{0}^{2 \pi} g^{k}\left(\mu_{0}, \phi\right) \varphi_{j}(\phi) \frac{\sqrt{\alpha \cos ^{2} \phi+\beta \sin ^{2} \phi}}{\sqrt{\alpha \beta}} d \phi,
\end{gathered}
$$




$$
\begin{aligned}
q_{i j}=-\frac{\sqrt{a b}}{R \pi} \sum_{m=0}^{\infty} \int_{0}^{2 \pi} \int_{0}^{2 \pi}\left[\frac{H e_{m}^{\prime}\left(\mu_{0}\right)}{H e_{m}\left(\mu_{0}\right)} c e_{m}(\phi, q) c e_{m}\left(\phi^{\prime}, q\right)\right. \\
\left.+\frac{H o_{m}^{\prime}\left(\mu_{0}\right)}{H o_{m}\left(\mu_{0}\right)} s e_{m}(\phi, q) s e_{m}\left(\phi^{\prime}, q\right)\right] \varphi_{i}(\phi) \varphi_{j}\left(\phi^{\prime}\right) d \phi^{\prime} d \phi
\end{aligned}
$$

The Equation (29) has a unique solution. After finding $u_{0 j}^{k}(j=1,2, \ldots, N)$, we can obtain

$$
\begin{aligned}
u_{h}^{k}(\mu, \phi)= & \frac{1}{\pi} \sum_{j=1}^{N} u_{0 j}^{k}\left[\sum _ { m = 0 } ^ { \infty } \int _ { 0 } ^ { 2 \pi } \left(\frac{H e_{m}(\mu)}{H e_{m}\left(\mu_{0}\right)} c e_{m}(\phi, q) c e_{m}\left(\phi^{\prime}, q\right)\right.\right. \\
& \left.\left.+\frac{H o_{m}(\mu)}{H o_{m}\left(\mu_{0}\right)} \operatorname{se}_{m}(\phi, q) s e_{m}\left(\phi^{\prime}, q\right)\right) \varphi_{j}\left(\phi^{\prime}\right) d \phi^{\prime}\right], \mu>\mu_{0} .
\end{aligned}
$$

As an example, we shall give the formulae of $q_{i j}$ by using piecewise linear interpolation below. we take piecewise linear interpolation under the uniform subdivision

$$
L_{i}(\phi)= \begin{cases}\frac{N}{2 \pi}\left(\phi-\phi_{i-1}\right), & \phi \in\left[\phi_{i-1}, \phi_{i}\right], \\ \frac{N}{2 \pi}\left(\phi_{i+1}-\phi\right), & \phi \in\left[\phi_{i}, \phi_{i+1}\right], \\ 0, \text { otherwise, } & \end{cases}
$$

where $i=1,2, \ldots, N, \phi_{i}=\frac{i}{N} 2 \pi$, then $L_{i}\left(\phi_{j}\right)=\delta_{i}^{j}, i, j=1,2, \ldots, N$,

$$
\sum_{i=1}^{N} L_{i}(\phi)=1, \text { and } \operatorname{span}\left\{L_{i}(\phi)\right\}_{i=1}^{N} \subset H^{\frac{1}{2}}(\widetilde{\Gamma}) .
$$

It is not difficult to get the following

$$
\begin{aligned}
q_{i j}= & -\frac{\sqrt{a b}}{R \pi} \sum_{m=0}^{\infty}\left[\frac{H e_{2 m}^{\prime}\left(\mu_{0}\right)}{H e_{2 m}\left(\mu_{0}\right)} p_{i}(2 m) p_{j}(2 m)+\frac{H e_{2 m+1}^{\prime}\left(\mu_{0}\right)}{H e_{2 m+1}\left(\mu_{0}\right)} P_{i}(2 m+1) P_{j}(2 m+1)\right. \\
& \left.+\frac{H o_{2 m+1}^{\prime}\left(\mu_{0}\right)}{H o_{2 m+1}\left(\mu_{0}\right)} s_{i}(2 m+1) s_{j}(2 m+1)+\frac{H o_{2 m+2}^{\prime}\left(\mu_{0}\right)}{H o_{2 m+2}\left(\mu_{0}\right)} S_{i}(2 m+2) S_{j}(2 m+2)\right],
\end{aligned}
$$

and the approximate solution is

$$
\begin{aligned}
u_{h}^{k}= & \frac{1}{\pi} \sum_{j=1}^{N} u_{0 j}^{k}\left\{\sum_{m=0}^{\infty}\left[\left(\sum_{l=0}^{\infty} A_{2 l}^{(2 m)} \cos (2 l \phi)\right) \frac{H e_{2 m}(\mu)}{H e_{2 m}\left(\mu_{0}\right)} p_{j}(2 m)\right]\right. \\
& +\sum_{m=0}^{\infty}\left[\left(\sum_{l=0}^{\infty} A_{2 l}^{(2 m+1)} \cos ((2 l+1) \phi)\right) \frac{H e_{2 m+1}(\mu)}{H e_{2 m+1}\left(\mu_{0}\right)} P_{j}(2 m+1)\right] \\
& +\sum_{m=0}^{\infty}\left[\left(\sum_{l=0}^{\infty} B_{2 l}^{(2 m+1)} \sin ((2 l+1) \phi)\right) \frac{H o_{2 m+1}(\mu)}{H o_{2 m+1}\left(\mu_{0}\right)} s_{j}(2 m+1)\right] \\
& \left.+\sum_{m=0}^{\infty}\left[\left(\sum_{l=0}^{\infty} B_{2 l}^{(2 m+2)} \sin ((2 l+2) \phi)\right) \frac{H o_{2 m+2}(\mu)}{H o_{2 m+2}\left(\mu_{0}\right)} S_{j}(2 m+2)\right]\right\}
\end{aligned}
$$

where

$$
\begin{gathered}
p_{j}(2 m)=A_{0}^{(2 m)} \frac{2 \pi}{N}+\sum_{l=1}^{\infty} A_{2 l}^{(2 m)} \frac{2 N}{\pi(2 l)^{2}} \sin ^{2}\left(\frac{2 l \pi}{N}\right) \cos \left(\frac{4 j l \pi}{N}\right), \\
P_{j}(2 m+1)=\sum_{l=0}^{\infty} A_{2 l}^{(2 m+1)} \frac{2 N}{\pi(2 l+1)^{2}} \sin ^{2}\left(\frac{(2 l+1) \pi}{N}\right) \cos \left(\frac{2(2 l+1) j \pi}{N}\right), \\
s_{j}(2 m+1)=\sum_{l=0}^{\infty} B_{2 l}^{(2 m+1)} \frac{2 N}{\pi(2 l+1)^{2}} \sin ^{2}\left(\frac{(2 l+1) \pi}{N}\right) \sin \left(\frac{2(2 l+1) j \pi}{N}\right),
\end{gathered}
$$




$$
S_{j}(2 m+2)=\sum_{l=0}^{\infty} B_{2 l}^{(2 m+2)} \frac{2 N}{\pi(2 l+2)^{2}} \sin ^{2}\left(\frac{(2 l+2) \pi}{N}\right) \sin \left(\frac{2(2 l+2) j \pi}{N}\right) .
$$

From (33), we know

$$
q_{i j}=q_{j i}, \quad i, j=1,2, \cdots, N .
$$

(35) shows that the matrix $\boldsymbol{Q}$ is symmetric.

\subsection{Error Estimates}

Theorem 3.2 Let $u_{0}^{k}$ and $u_{0 h}^{k}$ be solutions of the variational problems (27), (28) respectively, and $S^{h}(\widetilde{\Gamma})$ be the space of piecewise polynomials which are of degree $j(j \geqslant 1), u_{0}^{k} \in H^{j+1}(\widetilde{\Gamma})$, then there exists a constant $C$ independent of $h$, the following inequality holds

$$
\left\|u_{0}^{k}-u_{0 h}^{k}\right\|_{L^{2}(\widetilde{\Gamma})} \leqslant C h^{j+1}\left|u_{0}^{k}\right|_{H^{j+1}(\widetilde{\Gamma})} .
$$

Theorem 3.3 Let $u^{k}$ and $u_{h}^{k}$ be solutions obtained by (25), (31) respectively, then there exists a constant $C(\mu, \phi)$ independent of $h$, the following inequality holds

$$
\left|u^{k}-u_{h}^{k}\right| \leqslant C(\mu, \phi)\left\|u_{0}^{k}-u_{0 h}^{k}\right\|_{L^{2}(\widetilde{\Gamma})} .
$$

\section{Numerical Examples}

To demonstrate the performance of this method, we consider the numerical solutions of the following initialboundary value problem:

$$
\begin{gathered}
\frac{\partial^{2} u}{\partial t^{2}}-\left(a \frac{\partial^{2} u}{\partial x^{2}}+b \frac{\partial^{2} u}{\partial y^{2}}\right)=0, \quad(x, y, t) \in \Omega^{c} \times I, \\
a n_{x} \frac{\partial u}{\partial x}+b n_{y} \frac{\partial u}{\partial y}=g(x, y, t),(x, y, t) \in \Gamma \times I, \\
u(x, y, 0)=u_{0}(x, y), \quad u_{t}(x, y, 0)=v_{0}(x, y), \quad(x, y) \in \Omega^{c} .
\end{gathered}
$$

Where $b>a>0, \Omega^{c}=\left\{(x, y) \mid \alpha x^{2}+\beta y^{2}>R^{2}\right\}, \Gamma=\left\{(x, y) \mid \alpha x^{2}+\beta y^{2}=R^{2}\right\}, \boldsymbol{n}=\left(n_{x}, n_{y}\right)=-\left(\frac{\alpha x}{\sqrt{\alpha^{2} x^{2}+\beta^{2} y^{2}}}, \frac{\beta y}{\sqrt{\alpha^{2} x^{2}+\beta^{2} y^{2}}}\right)$. Functions $g(x, y, t), u_{0}(x, y)$ and $v_{0}(x, y)$ are given as follows

$$
\begin{gathered}
g(x, y, t)=-\frac{1}{\sqrt{\alpha^{2} x^{2}+\beta^{2 y^{2}}}}\left[\left(\frac{\sqrt{a} x}{E(x, y)} H_{1}^{(1)}(\omega E(x, y))-\frac{\left(x^{3}+x y^{2}\right) \omega}{\sqrt{a} E^{2}(x, y)} H_{2}^{(1)}(\omega E(x, y))\right)\right. \\
\left.+i\left(\frac{\sqrt{b} y}{E(x, y)} H_{1}^{(1)}(\omega E(x, y))-\frac{\left(y^{3}+x^{2} y\right) \omega}{\sqrt{b} E^{2}(x, y)} H_{2}^{(1)}(\omega E(x, y))\right)\right](\cos (\omega t)+\sin (\omega t)) \\
u_{0}(x, y)=H_{1}^{(1)}(\omega E(x, y))\left(\frac{x}{\sqrt{a} E(x, y)}+i \frac{y}{\sqrt{b} E(x, y)}\right) \\
v_{0}(x, y)=\omega H_{1}^{(1)}(\omega E(x, y))\left(\frac{x}{\sqrt{a} E(x, y)}+i \frac{y}{\sqrt{b} E(x, y)}\right) .
\end{gathered}
$$

Here $H_{1}^{(1)}(z)$ and $H_{2}^{(1)}(z)$ are the Hankel functions of the first order, $E(x, y)=\sqrt{\frac{x^{2}}{a}+\frac{y^{2}}{b}}$. Furthermore, some conditions at infinity are needed. The exact solution of this problem is

$$
u(x, y, t)=H_{1}^{(1)}(\omega E(x, y))\left(\frac{x}{\sqrt{a} E(x, y)}+i \frac{y}{\sqrt{b} E(x, y)}\right)(\cos (\omega t)+\sin (\omega t)) .
$$

If we take $a=1, b=2, \alpha=1, \beta=2, R=0.9$, then $f_{0} \approx 0.77942, \mu_{0}=\ln \sqrt{3} . \sum_{m=0}^{\infty}$ and $\sum_{l=0}^{\infty}$ can be replaced by $\sum_{m=0}^{M}$ and $\sum_{l=0}^{L}$ respectively. $M$ and $L$ may be not large numbers, such as $M=20, L=10$ when $N=32$, while $M=40$, $L=20$ when $N=64$. Tables 4.1-4.4 give some values of numerical solution $u_{h}(\mu, \phi, t)$, exact solution $u(\mu, \phi, t)$ and relative error $E_{r}=\left|u-u_{h}\right| /|u|$. 
Table 4.1. Numerical solution $u_{h}$, exact solution $u$ and relative error $E_{r}(N=32, t=0.3, \omega=1)$

\begin{tabular}{cccccccc}
\hline \multirow{2}{*}{$\mu$} & \multirow{2}{*}{$\phi$} & \multicolumn{2}{c}{$\operatorname{Real}$ part of solution } & & \multicolumn{2}{c}{$\operatorname{Imaginary}$ part of solution } & \multirow{2}{*}{$E_{r}(\tau=0.05)$} \\
\cline { 3 - 4 } & & $\operatorname{Re}\left(u_{h}\right)$ & $\operatorname{Re}(u)$ & & $\operatorname{Im}\left(u_{h}\right)$ & $\operatorname{Im}(u)$ & \\
\hline 1.0 & 0 & $6.24156 \mathrm{e}-1$ & $6.24155 \mathrm{e}-1$ & & $-7.74424 \mathrm{e}-2$ & $-7.74425 \mathrm{e}-2$ & $1.12234 \mathrm{e}-6$ \\
1.5 & 0 & $7.27816 \mathrm{e}-1$ & $7.27815 \mathrm{e}-1$ & & $-2.54670 \mathrm{e}-1$ & $-2.54670 \mathrm{e}-1$ & $1.29504 \mathrm{e}-6$ \\
1.5 & $\pi / 8$ & $7.77075 \mathrm{e}-1$ & $7.77075 \mathrm{e}-1$ & & $-1.42319 \mathrm{e}-4$ & $-1.42499 \mathrm{e}-4$ & $1.03780 \mathrm{e}-6$ \\
3.0 & $\pi / 4$ & $3.57737 \mathrm{e}-1$ & $3.57737 \mathrm{e}-1$ & & $6.69196 \mathrm{e}-3$ & $6.69187 \mathrm{e}-3$ & $1.31773 \mathrm{e}-6$ \\
6.0 & 2 & $7.77940 \mathrm{e}-2$ & $7.77939 \mathrm{e}-2$ & & $-1.68462 \mathrm{e}-2$ & $-1.68462 \mathrm{e}-2$ & $1.32230 \mathrm{e}-6$ \\
\hline
\end{tabular}

Table 4.2. Numerical solution $u_{h}$, exact solution $u$ and relative error $E_{r}(N=32, t=0.5, \omega=1)$

\begin{tabular}{cccccccc}
\hline \multirow{2}{*}{$\mu$} & \multirow{2}{*}{$\phi$} & \multicolumn{2}{c}{$\operatorname{Real}$ part of solution } & & \multicolumn{2}{c}{$\operatorname{Imaginary}$ part of solution } & \multirow{2}{*}{$E_{r}(\tau=0.025)$} \\
\cline { 3 - 4 } & & $\operatorname{Re}\left(u_{h}\right)$ & $\operatorname{Re}(u)$ & & $\operatorname{Im}\left(u_{h}\right)$ & $\operatorname{Im}(u)$ & \\
\hline 1.0 & 0 & $6.77123 \mathrm{e}-1$ & $6.77122 \mathrm{e}-1$ & & $-8.40144 \mathrm{e}-1$ & $-8.40145 \mathrm{e}-1$ & $1.12234 \mathrm{e}-6$ \\
1.5 & 0 & $7.89581 \mathrm{e}-1$ & $7.89579 \mathrm{e}-1$ & & $-2.76282 \mathrm{e}-1$ & $-2.76282 \mathrm{e}-1$ & $1.29504 \mathrm{e}-6$ \\
1.5 & $\pi / 8$ & $8.43020 \mathrm{e}-1$ & $8.43019 \mathrm{e}-1$ & & $-1.54396 \mathrm{e}-4$ & $-1.54592 \mathrm{e}-4$ & $1.03780 \mathrm{e}-6$ \\
3.0 & $\pi / 4$ & $3.88096 \mathrm{e}-1$ & $3.88095 \mathrm{e}-1$ & & $7.25986 \mathrm{e}-3$ & $7.25976 \mathrm{e}-3$ & $1.31773 \mathrm{e}-6$ \\
6.0 & 2 & $8.43958 \mathrm{e}-2$ & $8.43957 \mathrm{e}-2$ & & $-1.82758 \mathrm{e}-2$ & $-1.82758 \mathrm{e}-2$ & $1.32230 \mathrm{e}-6$ \\
\hline
\end{tabular}

Table 4.3. Numerical solution $u_{h}$, exact solution $u$ and relative error $E_{r}(N=64, t=0.5, \omega=1)$

\begin{tabular}{cccccccc}
\hline \multirow{2}{*}{$\mu$} & \multirow{2}{*}{$\phi$} & \multicolumn{2}{c}{$\operatorname{Real}$ part of solution } & & \multicolumn{2}{c}{$\operatorname{Imaginary}$ part of solution } & \multirow{2}{*}{$E_{r}(\tau=0.05)$} \\
\cline { 3 - 4 } & & $\operatorname{Re}\left(u_{h}\right)$ & $\operatorname{Re}(u)$ & & $\operatorname{Im}\left(u_{h}\right)$ & $\operatorname{Im}(u)$ & \\
\hline 1.0 & 0 & $6.77123 \mathrm{e}-1$ & $6.77122 \mathrm{e}-1$ & & $-8.40145 \mathrm{e}-1$ & $-8.40145 \mathrm{e}-1$ & $2.19990 \mathrm{e}-7$ \\
1.5 & 0 & $7.89580 \mathrm{e}-1$ & $7.89579 \mathrm{e}-1$ & & $-2.76282 \mathrm{e}-1$ & $-2.76282 \mathrm{e}-1$ & $3.13724 \mathrm{e}-7$ \\
1.5 & $\pi / 8$ & $8.43020 \mathrm{e}-1$ & $8.43019 \mathrm{e}-1$ & & $-1.54533 \mathrm{e}-4$ & $-1.54592 \mathrm{e}-4$ & $2.59550 \mathrm{e}-7$ \\
3.0 & $\pi / 4$ & $3.88096 \mathrm{e}-1$ & $3.88095 \mathrm{e}-1$ & & $7.25979 \mathrm{e}-3$ & $7.25976 \mathrm{e}-3$ & $3.37652 \mathrm{e}-7$ \\
6.0 & 2 & $8.43957 \mathrm{e}-2$ & $8.43957 \mathrm{e}-2$ & & $-1.82758 \mathrm{e}-2$ & $-1.82758 \mathrm{e}-2$ & $3.37347 \mathrm{e}-7$ \\
\hline
\end{tabular}

Table 4.4. Numerical solution $u_{h}$, exact solution $u$ and relative error $E_{r}(N=64, t=0.5, \omega=1)$

\begin{tabular}{cccccccc}
\hline \multirow{2}{*}{$\mu$} & \multirow{2}{*}{$\phi$} & \multicolumn{2}{c}{$\operatorname{Real}$ part of solution } & & \multicolumn{2}{c}{$\operatorname{Imaginary}$ part of solution } & \multirow{2}{*}{$E_{r}(\tau=0.025)$} \\
& & $\operatorname{Re}\left(u_{h}\right)$ & $\operatorname{Re}(u)$ & & $\operatorname{Im}\left(u_{h}\right)$ & $\operatorname{Im}(u)$ & \\
\hline 1.0 & 0 & $6.77123 \mathrm{e}-1$ & $6.77122 \mathrm{e}-1$ & & $-8.40145 \mathrm{e}-1$ & $-8.40145 \mathrm{e}-1$ & $2.19990 \mathrm{e}-7$ \\
1.5 & 0 & $7.89580 \mathrm{e}-1$ & $7.89579 \mathrm{e}-1$ & & $-2.76282 \mathrm{e}-1$ & $-2.76282 \mathrm{e}-1$ & $3.13724 \mathrm{e}-7$ \\
1.5 & $\pi / 8$ & $8.43020 \mathrm{e}-1$ & $8.43019 \mathrm{e}-1$ & & $-1.54533 \mathrm{e}-4$ & $-1.54592 \mathrm{e}-4$ & $2.59550 \mathrm{e}-7$ \\
3.0 & $\pi / 4$ & $3.88096 \mathrm{e}-1$ & $3.88095 \mathrm{e}-1$ & & $7.25979 \mathrm{e}-3$ & $7.25976 \mathrm{e}-3$ & $3.37652 \mathrm{e}-7$ \\
6.0 & 2 & $8.43957 \mathrm{e}-2$ & $8.43957 \mathrm{e}-2$ & & $-1.82758 \mathrm{e}-2$ & $-1.82758 \mathrm{e}-2$ & $3.37347 \mathrm{e}-7$ \\
\hline
\end{tabular}

The curves of errors are partly depicted as follows.
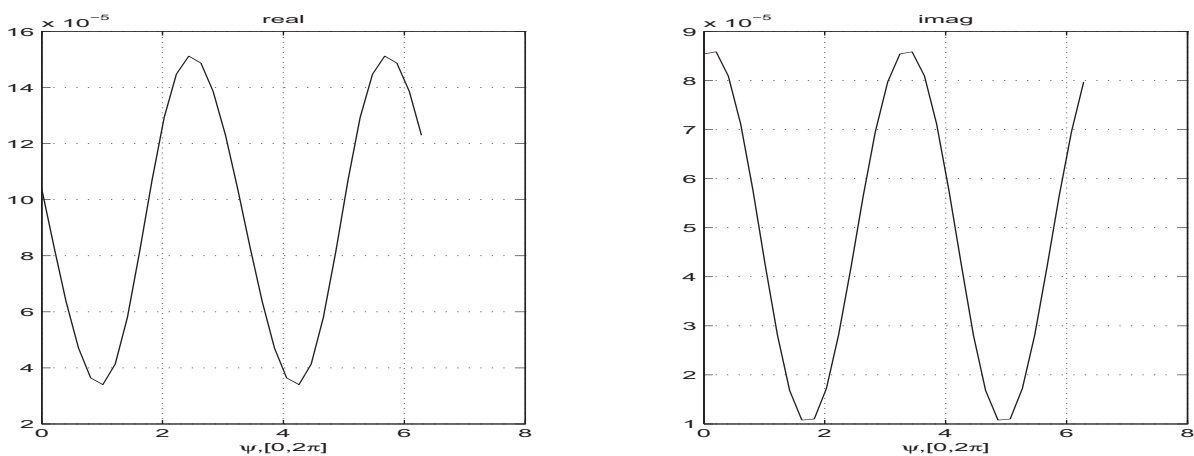

Figure 4.1. $\mu=2, \omega=1, t=0.3, \tau=0.05, T=1, N=32, M=20, L=10$, the step of $\phi$ is $\frac{2 \pi}{N}$. The curve of the relative error for all $\phi \in[0,2 \pi]$. left: $\operatorname{Re}\left(\frac{u-u^{h}}{u}\right)$, right: $\operatorname{Im}\left(\frac{u-u^{h}}{u}\right)$ 

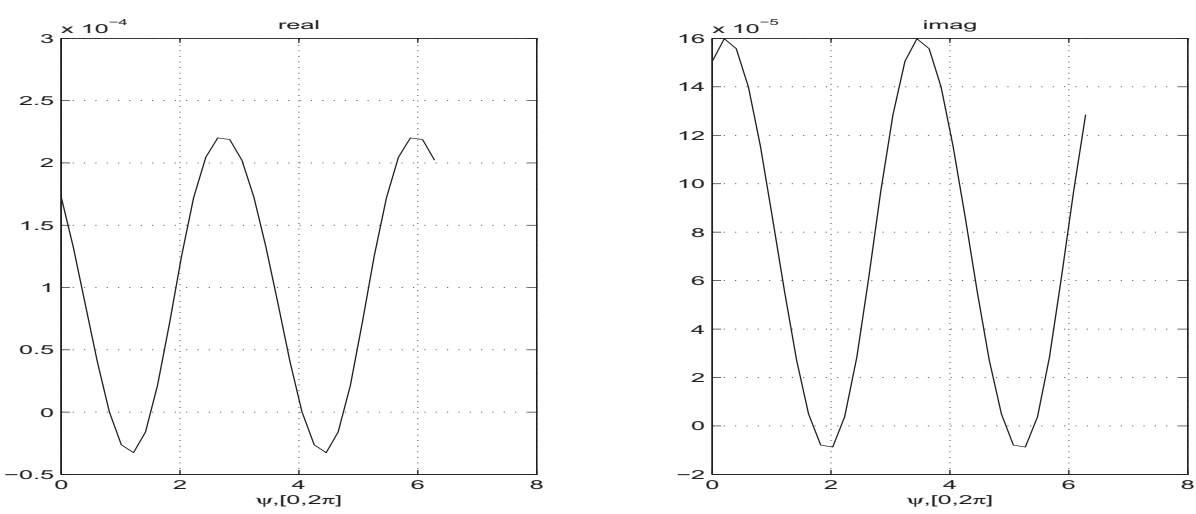

Figure 4.2. $\mu=2, \omega=1.5, t=0.3, \tau=0.05, T=1, N=32, M=20, L=10$, the step of $\phi$ is $\frac{2 \pi}{N}$. The curve of the relative error for all $\phi \in[0,2 \pi]$. left: $\operatorname{Re}\left(\frac{u-u^{h}}{u}\right)$, right: $\operatorname{Im}\left(\frac{u-u^{h}}{u}\right)$
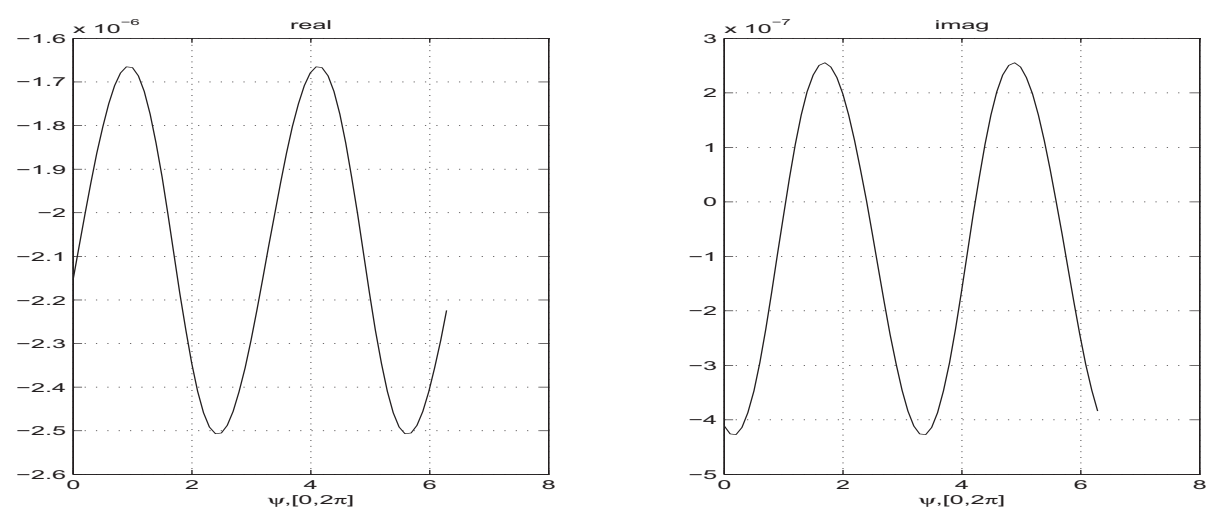

Figure 4.3. $\mu=2, \omega=1, t=0.3, \tau=0.05, T=1, N=64, M=40, L=20$, the step of $\phi$ is $\frac{2 \pi}{N}$. The curve of the relative error for all $\phi \in[0,2 \pi]$. left: $\operatorname{Re}\left(\frac{u-u^{h}}{u}\right)$, right: $\operatorname{Im}\left(\frac{u-u^{h}}{u}\right)$
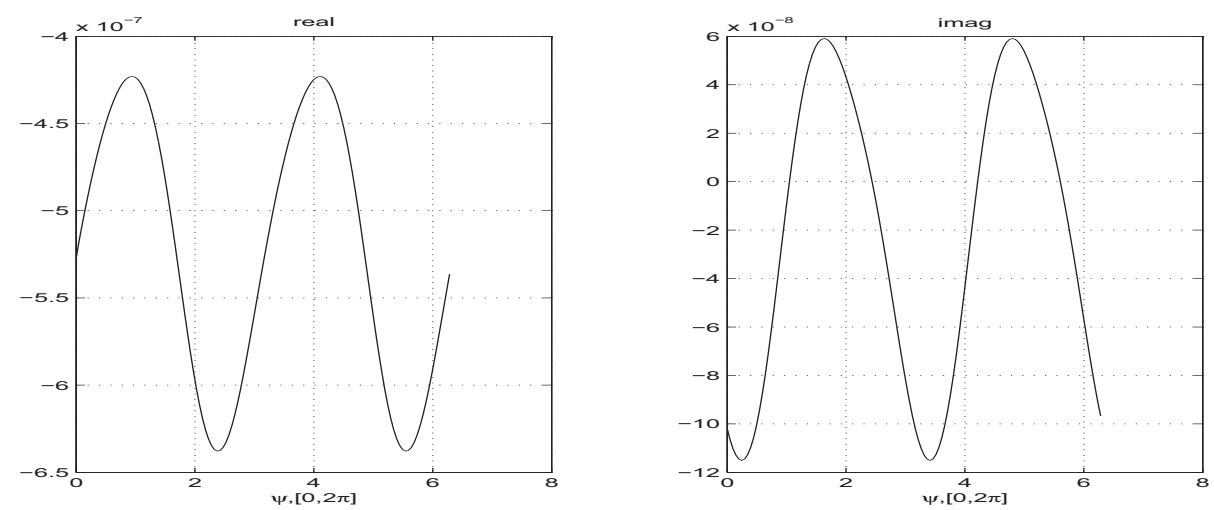

Figure 4.4. $\mu=2, \omega=1, t=0.3, \tau=0.05, T=1, N=128, M=80, L=40$, the step of $\phi$ is $\frac{2 \pi}{N}$. The curve of the relative error for all $\phi \in[0,2 \pi]$. left: $\operatorname{Re}\left(\frac{u-u^{h}}{u}\right)$, right: $\operatorname{Im}\left(\frac{u-u^{h}}{u}\right)$ 

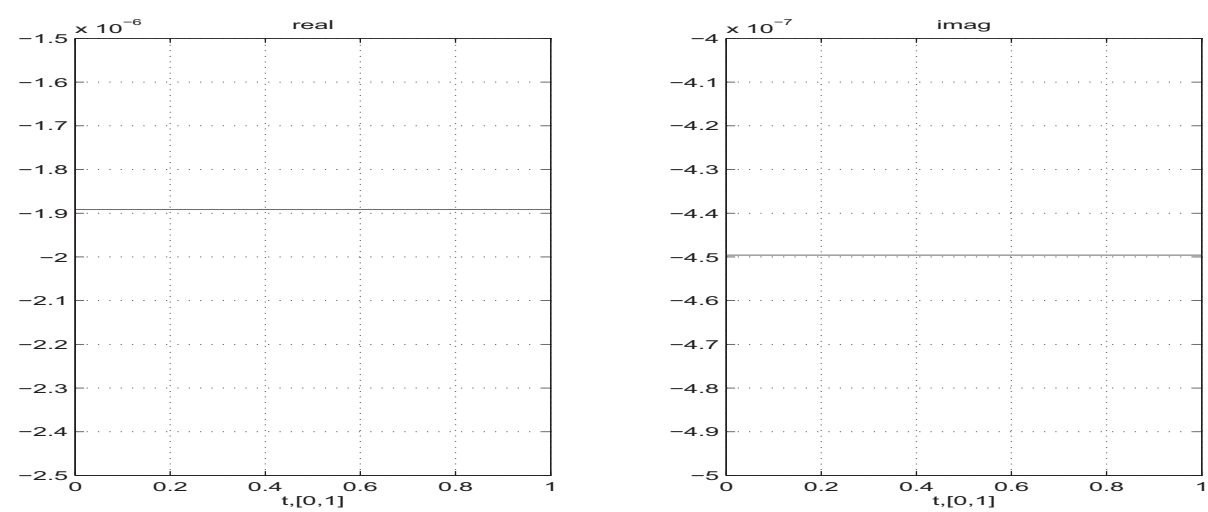

Figure 4.5. $\mu=2, \omega=1.5, \phi=0$. Taking $\tau=0.05, N=32, M=20, L=10, T=1$, the curve of the relative error for all $t \in[0,1]$. left: $\operatorname{Re}\left(\frac{u-u^{h}}{u}\right)$, right: $\operatorname{Im}\left(\frac{u-u^{h}}{u}\right)$
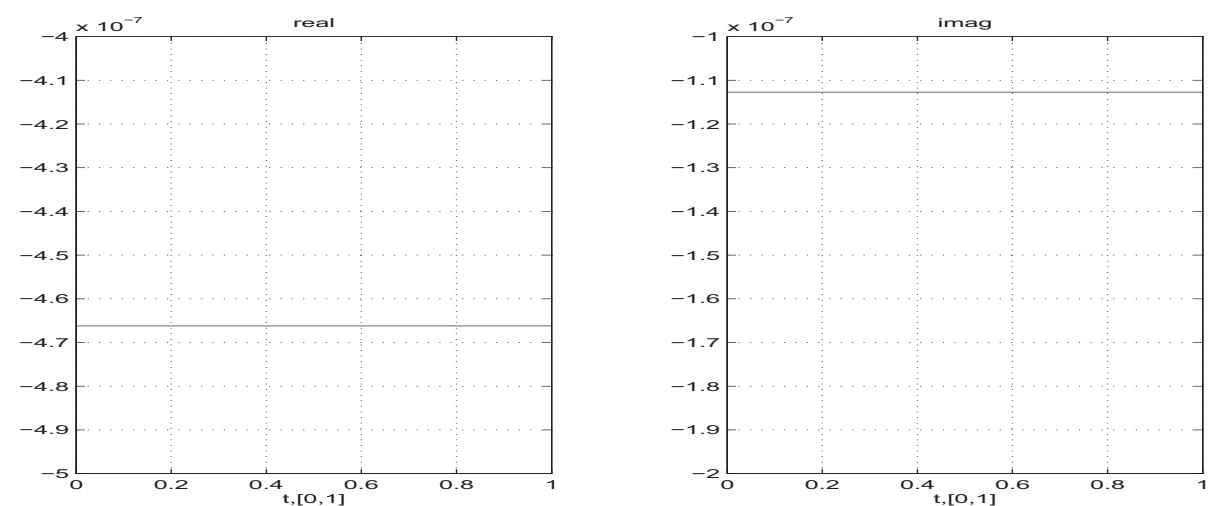

Figure 4.6. $\mu=2, \omega=1.5, \phi=0$. Taking $\tau=0.025, N=32, M=20, L=10, T=1$, the curve of the relative error for all $t \in[0,1]$. left: $\operatorname{Re}\left(\frac{u-u^{h}}{u}\right)$, right: $\operatorname{Im}\left(\frac{u-u^{h}}{u}\right)$
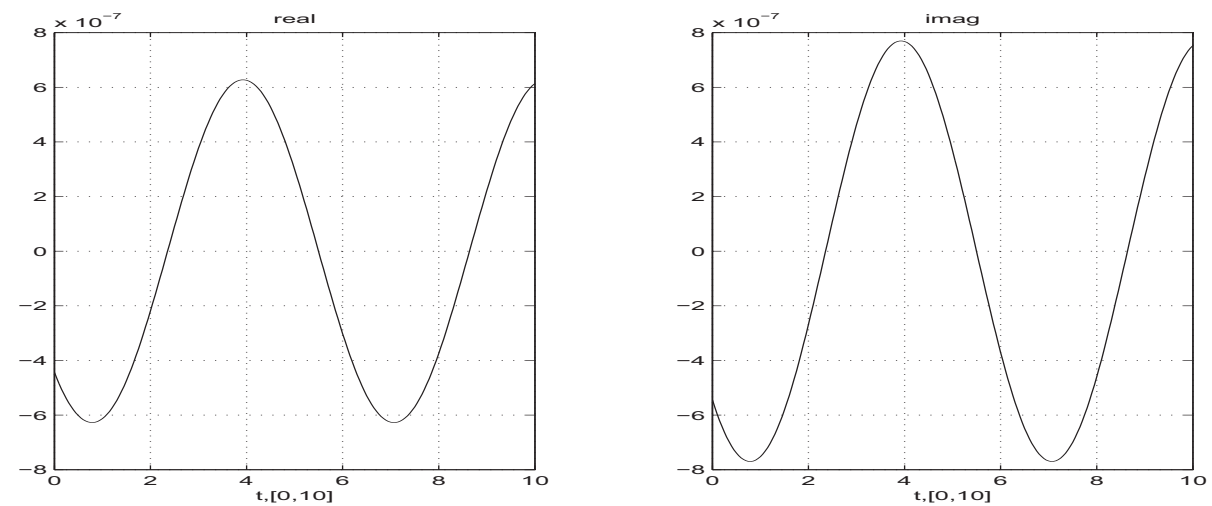

Figure 4.7. $\mu=2, \omega=1, \phi=0$. Taking $\tau=0.05, N=32, M=20, L=10, T=10$, the curve of the absolute error for all $t \in[0,10]$. left: $\operatorname{Re}\left(u-u^{h}\right)$, right: $\operatorname{Im}\left(u-u^{h}\right)$ 

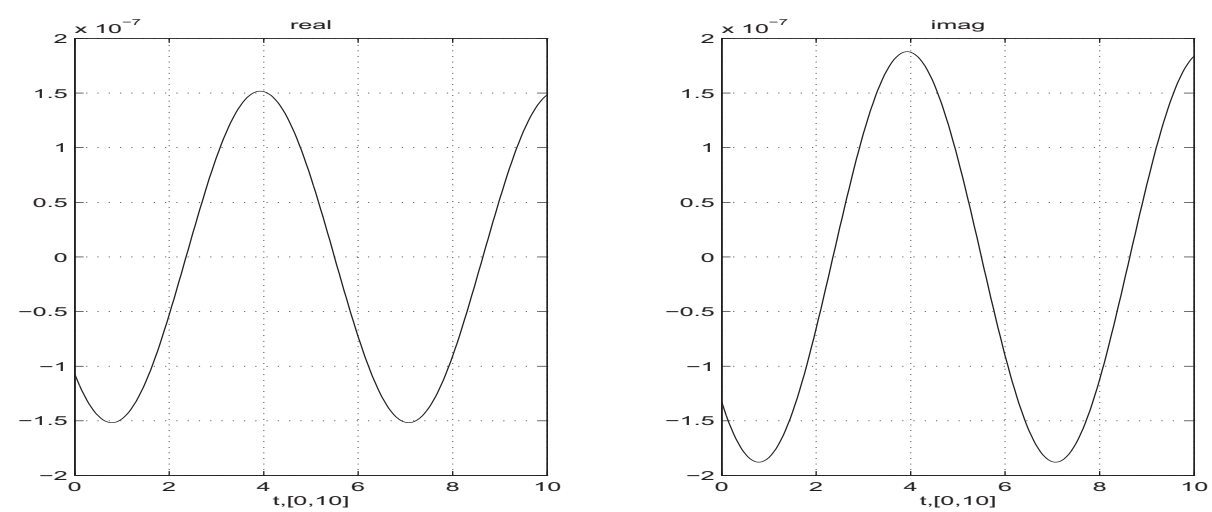

Figure 4.8. $\mu=2, \omega=1, \phi=0$. Taking $\tau=0.05, N=64, M=40, L=20, T=10$, the curve of the absolute error for all $t \in[0,10]$. left: $\operatorname{Re}\left(u-u^{h}\right)$, right: $\operatorname{Im}\left(u-u^{h}\right)$

\section{Conclusions}

In this paper, the natural boundary element method for an anisotropic hyperbolic problem in an exterior elliptic domain is investigated. By introducing the elliptic coordinates and the separation of variables, the original problem is turned into exterior Helmholtz problem with elliptic boundary which is equivalent to the original problem. We mainly study the solutions of the Poisson integral formula and the natural integral equation. Since the Poisson integral formula and the natural integral equation involve the computation of the special functions-the Mathieu functions and the modified Mathieu functions, to solve them numerically we use the method in Zhang and Du (2008) and Gutiérrez-Vega and Podríguez-Dagnino (2003). Numerical results demonstrate the feasibility and efficiency of this method.

\section{Acknowledgements}

This research was partly supported by the National Natural Science Foundation of China, contact/grant number 10871100, 11371198 and 11071109. The computation in this paper has been carried out in the Jiangsu Provincial Key Laboratory for Numerical Simulation of Large Scale Complex Systems. The authors express their thanks to them.

\section{References}

Ben-Poart, G., \& Givoli, D. (1995). Solution of unbounded domain problems using elliptic artificial boundaries. Communication in Numerical Methods in Engineering, 11, 735-741. http://dx.doi.org/10.1002/cnm.1640110904

Du, Q. K., \& Yu, D. H. (1999). The natural integral equation for initial boundary value problems of parabolic equation and its numerical implementation. Math. Numer. Sinica, 21(4), 495-506 (in Chinese).

Du, Q. K., \& Yu, D. H. (2001). On the natural integral equation for initial boundary value problems of two dimensional hyperbolic equation. Acta Math. Appl. Sinica, 24(1), 17-26 (in Cineses).

Feng, K. (1980). Differential vs. integral equations and finite vs. infinite elements. Math. Num. Sinica, 2(1), 100-105 (in Chinese).

Feng, K. (1983). Finite element method and natural boundary reduction. Proceedings of the International Congress Mathematicians (pp. 1439-1453). Warszawa: Polish Academy Press.

Hao, P., \& Du, Q. K. (2009). Natural boundary element method for a kind of anisotropic parabolic problem in an exterior domain. Appl. Math. J. Chinese Univ., 24(3), 368-378 (in Chinese).

Wu, J. M., \& Yu, D. H. (2000). The natural boundary element method for exterior elliptic domain. Math. Numer. Sinica, 22(3), 355-368 (in Chinese).

Yu, D. H. (1993). Mathematical Theory of Natural Boundary Element Method (in Chinese). Beijing: Science Press.

Yu, D. H. (2002). Natural Boundary Integral Method and Its Applications. Science Press \& Kluwer Academic Publishers. 
Yu, D. H., \& Du, Q. K. (2003). The coupling of natural boundary element and finite element method for 2D hyperbolic equations. J. Comput. Math., 21(5), 583-594.

Zhang, M., \& Du, Q. K. (2008). Natural boundary element method for the Helmholtz problems in an exterior elliptic domain. Math. Numer. Sinica, 30(1), 75-88 (in Chinese).

Zhu, W., \& Du, Q. K. (2004). The overlapping domain decomposition method for an anisotropic exterior elliptic problem. Math. Numer. Sinica, 26(4), 459-472 (in Chinese).

\section{Copyrights}

Copyright for this article is retained by the author(s), with first publication rights granted to the journal.

This is an open-access article distributed under the terms and conditions of the Creative Commons Attribution license (http://creativecommons.org/licenses/by/3.0/). 